\title{
Gegen das Altern hilft auch kein Testosteron
}

\author{
Die Substitution von Testosteron bei Männern wird intensiv beworben und immer häufiger \\ praktiziert - bringt aber keine Vorteile. Drei aktuelle Studien beleuchten die Situation.
}

_ Für eine Studie wurden 493 Patienten im Alter $>65$ Jahren mit niedrigen Testosteronwerten und kognitiven Störungen in eine Testosteron-Gel- und eine Placebogruppe randomisiert. Die Testosterondosis wurde so eingestellt, dass die Plasmaspiegel den Normalwerten von 19- bis 40-jährigen Männern entsprachen, also 500-800 ng/dl. Nach 6-12 Monaten zeigten bei zahlreichen kognitiven Prüfungen keine Unterschiede zwischen den Gruppen [1].

In einer methodisch vergleichbaren randomisierten Studie wurden 170 Probanden jenseits des 65 . Lebensjahrs mit niedrigen Testosteronausgangswerten per CT untersucht. Unter Testosterongabe zeigte sich innerhalb eines Jahres eine signifikant stärkere Zunahme nicht-kalzifizierter Koronarplaques [2].
Eine dritte Studie untersuchte über fünf Jahre die Korrelation zwischen der in den USA erlaubten TV-Werbung für Testosteron und den Verordnungen. Monatlich wurden im Mittel 13 Spots gesendet. Von 17 Millionen versicherten Zuschauern ließen 1.007.990 ihren Testosteronwert bestimmen. 283.317 erhielten eine Hormonverordnung, viele sogar ohne Laboruntersuchung. Diese Maßnahmen korrelierten signifikant mit der Zahl der Werbespots [3].

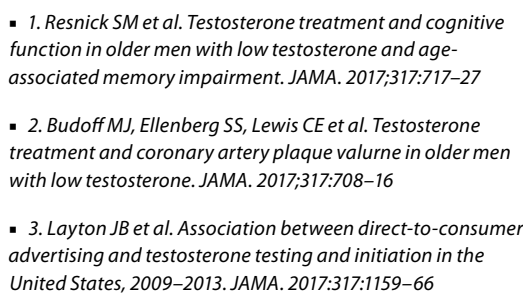

- 1. Resnick SM et al. Testosterone treatment and cognitive function in older men with low testosterone and ageassociated memory impairment. JAMA. 2017;317:717-27

- 2. BudoffMJ, Ellenberg SS, Lewis CE et al. Testosterone treatment and coronary artery plaque valurne in older men with low testosterone. JAMA. 2017;317:708-16

- 3. Layton JB et al. Association between direct-to-consumer advertising and testosterone testing and initiation in the United States, 2009-2013. JAMA. 2017:317:1159-66

\section{KOMMENTAR}

Die Substitution von Testosteron bei altersbedingt niedrigen Spiegeln zeigt in Studien eindeutig keine positiven Wirkungen auf körperliche und geistige Funktionen - und ist möglicherweise sogar kreislaufschädlich. Die FDA hat schon 2015 betont, dass sie hier keine Indikation sieht. Die Substitution erfreut sich bei Männern trotzdem zunehmender Beliebtheit. Da wird dann die Abnahme der Sexualfunktion im Alter als Hypogonadismus etikettiert. Folgen des Alterns müssen korrigiert, die Leistungsfähigkeit und die Lebenserwartung verbessert werden. Die Werbung verspricht den Testosteron-Jungbrunnen, die $\mathrm{Pa}$ tienten hören auf die Werbung - und die Ärzte hören auf ihre Patienten.

Prof. Dr. med. H. Holzgreve

\section{Dramatische Bergung eines Glassplitters aus dem Zökum}
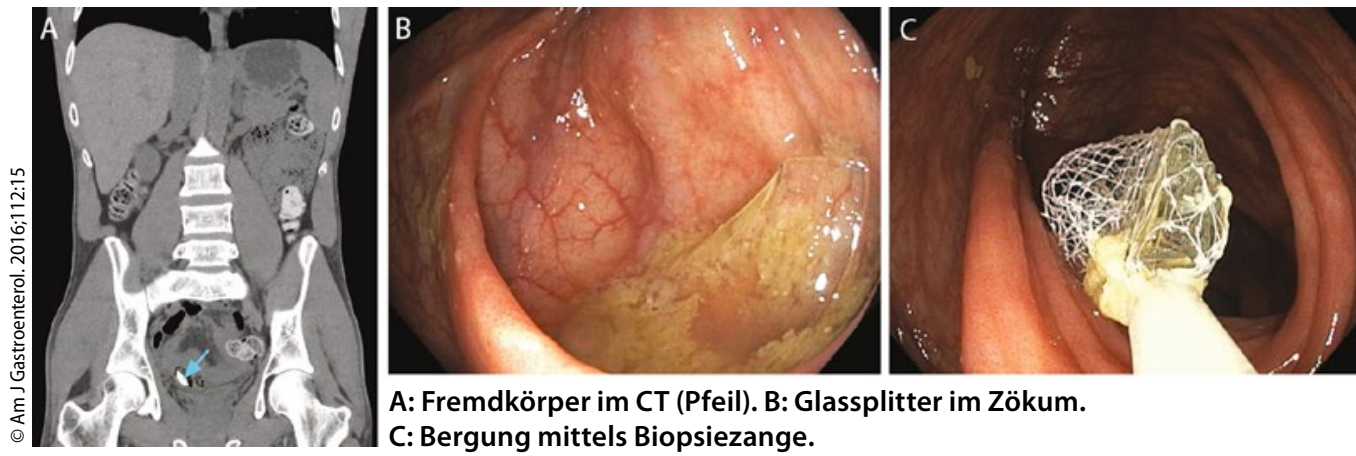

A: Fremdkörper im CT (Pfeil). B: Glassplitter im Zökum.

$\mathrm{C}$ : Bergung mittels Biopsiezange.

Ein 27-jähriger stellte sich mit Bauchschmerzen vor. Drei Tage zuvor hatte er bei einem Arbeitsunfall Glassplitter verschluckt. Auf dem Abdomen-CT erkannte man einen röntgendichten, polygonalen, ca. 1,5 cm großen Fremdkörper im Bereich des Zökums (Abb.

A). Zeichen für eine Perforation oder Blutung lagen nicht vor.

Am nächsten Tag wurde unter überwachter Anästhesie eine Koloskopie durchgeführt. Wie erwartet zeigte sich ein scharfer Glassplitter im Zökum (Abb. B). Mithilfe eines Bergungsnetzes und der Biopsiezange gelang es, den Glassplitter unter ständiger visueller Kontrolle im Zentrum des Lumens zu halten und das Objekt ohne Verletzung der Mukosa zu bergen (Abb. C). Ein kitzliger Moment war die Passage des Rektums, doch auch hier konnten durch vorsichtige Manipulation Verletzungen vermieden werden. Der Patient wurde beschwerdefrei entlassen. Die technische Leistung auf dem letzten Meter ist beeindruckend. Fast noch unfassbarer ist, dass dieser messerscharfe Fremdkörper auf dem deutlich längeren und turbulenteren Weg bis ins Zökum keine Schleimhautverletzungen verursacht hat.

Prof. Dr. med. H. S. FüeßI

- Elhanafi S et al. Endoscopic removal of shattered glass from the cecum. Am J Gastroenterol. 2016;112:15 